Reviews in Digital Humanities • Vol. 2, No. 4

\title{
Review: Readux
}

\section{James Lacitignola ${ }^{\mathbf{1}}$}

${ }^{1}$ University of Texas at Austin

Published on: Apr 12, 2021

License: Creative Commons Attribution 4.0 International License (CC-BY 4.0). 


\section{Project}

Readux

\section{Project Director}

$\underline{\text { Rebecca Sutton Koeser }}$

\section{Project URL}

https://readux.ecds.emory.edu/

\section{Project Reviewer}

James Lacitignola, University of Texas at Austin

\section{Project Overview}

\section{Rebecca Sutton Koeser, Jesse Karlsberg, and Sara Palmer}

Readux is an open source software platform developed at Emory University to offer a web interface for browsing, searching, and taking notes on collections of digitized print materials. The software also provides a user-friendly way to publish annotated critical editions of works as standalone websites.

Readux was originally developed from 2014 to 2016 with two major goals. First, Readux improves access to locally digitized print materials, which were previously only available in PDF form through the library catalog. Second, Readux enables the creation of richly annotated scholarly editions of multimodal printed works like the

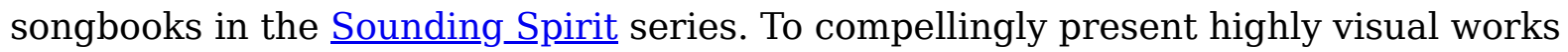
that include music notation or illustrations alongside textual content, Readux starts with digitized page images rather than encoded text or music, retaining the bibliographic form and design of the original.

Readux is built in Python/Django and based on the Fedora Commons 3 repository. Annotation functionality was implemented with annotator.js and required writing custom plugins to support rich text formatting, displaying annotations in the margin, and selecting and annotating portions of images. For volumes with page-level access, positional OCR text is converted into TEI facsimile, which is used to transparently overlay text on page images for selection and annotation. All the software developed for Readux is tested, documented, open source, and available on GitHub. What's more, the innovative software development for Readux eventually resulted in two spinoffs from the main codebase: piffle, a python library for working with International Image 
Interoperability Framework (IIIF) images, and django-annotator-store, a generalized annotator.js backend. A full list of the software developed is available on the site.

Scholarly edition export functionality was implemented with Jekyll, creating a static site which can be hosted on GitHub Pages for a simple and cheap publication solution. This approach was based on the conviction that scholars should not have their work locked into a proprietary system but should be able to take their editions with them and manage their own content.

Readux was built as a collaboration between the Library Software Engineering Team (LSET), the Emory Center for Digital Scholarship (ECDS), the Robert W. Woodruff Library, and the Pitts Theology Library. At times, the large number of contributors led to conflicts over ownership, investment, and vision for the project. LSET developed and managed the codebase-Rebecca Koeser served as technical lead and led primary development, Kevin Glover developed site design and annotation functionality, and Yang Li contributed to export functionality. ECDS staff crafted user stories, conducted product testing, and consulted at all levels of development-Alice Hickcox and Sara Palmer served as product managers and Jesse P. Karlsberg consulted on annotation and critical edition functionality.

Readux was funded in part by a grant from the Andrew W. Mellon Foundation. It attracted a collaborator at Wayne State University Libraries- Graham Hukill contributed code and set up a Readux instance to support work with their materials. Project contributors have presented on Readux at numerous digital humanities, library, and open repository conferences. A full list of project collaborators, citations, and presentations is available on the site credits page. The success of Readux inspired the ongoing development of a new version of the platform based on IIIF APIs instead of Fedora in order to support the display of interinstitutional thematic collections.

\section{Project Review}

\section{James Lacitignola}

Readux offers a web-based environment to read, analyze, annotate, and publish digital editions based on digital collections held by Emory University (Readux 1.8) and partner collections including the Sounding Spirit Project at UNC Press and the Folger Shakespeare folios project at Wayne State University (Readux 2.0). Currently, the 
Readux 1.8 corpus includes nineteen different collections, including 19th century Black literature and travel guides, Atlanta city guides, emblem books from Northern Europe, 19th century British popular novels known as "yellowbacks," and documents from the State of Georgia. The combination of creating annotations and publishing those annotated documents allows users to do close readings of archival materials. So often in the humanities we only see polished published works, and never the research process. By publishing their annotations, Readux users are able to document their analysis of individual documents that led them to make certain arguments. This makes more of the research process available for commentary from outside scholars, expanding collaborative research opportunities.

The process of using Readux is straight-forward with both video and written instructions available to new users. Users log into Readux using a Google, Emory, or social media account, then choose a document to view online, highlight the area they want to annotate, and type a note. Once the annotations have been made, Readux also allows users to export those documents and annotations, and publish them as a website on GitHub. The interface gives users incredible freedom to add their annotations over specific text or over entire sections of the page. This allows users the ability to comment on any aspects of a digitized document regardless of the type of object being annotated.

Readux is a clear example of how digital tools can streamline the sometimescumbersome process of conducting research in the humanities. Digital humanities scholars might find the ability to fork the Django-based software that is available on GitHub particularly useful. Deploying Readux to demonstrate how students can use annotations in research processes would also extend its utility outside of Emory's holdings. 